\title{
Rahmenbedingungen für eine digitale Gesundheitsversorgung aktiv gestalten.
}

\section{Der Schutz von Patientendaten ist nicht verhandelbar!}

Die digitale Transformation hat mittlerweile auch die Gesundheitsversorgung erreicht. Gesundheits-Apps, elektronische $\mathrm{Pa}$ tientenakten, telemedizinische Anwendungen und digitale ärztliche Kommunikation in Echtzeit sind längst technisch verfügbar und werden genutzt. Damit steigen jedoch die Risiken für einen sicheren und vertraulichen Umgang mit Patientendaten. Ereignisse wie der Anfang Januar 2019 bekannt gewordene Hackerangriff auf Daten von Politikern und Prominenten oder Berichte über eine Zunahme des Handels mit Gesundheitsdaten im sog. Darknet belegen dies.

Die Digitalisierung des Gesundheitswesens wird trotz dieser vielfältigen Gefährdungen weitergehen. Vor diesem Hintergrund stehen die Gesellschaft als Ganzes, vor allem aber die Politik und die Akteure der Selbstverwaltung im Gesundheitswesen in der Verantwortung, die Rahmenbedingungen für eine digitalisierte Gesundheitsversorgung aktiv zu gestalten und insbesondere zeitnah geeignete und wirksame Vorkehrungen zu einem angemessenen Schutz von Patientendaten im digitalisierten Zeitalter zu treffen. Eine einseitige Lockerung oder Streichung datenschutzrechtlicher Standards wäre dabei der falsche Weg.

Die mit der Digitalisierung des Gesundheitswesens verbundenen Chancen wie eine bessere Gesundheitsversorgung der Bevölkerung oder eine Reduzierung der Versorgungskosten sind unumstritten. Dies gilt allerdings auch für die damit zusammenhängenden Risiken.

Die digitale Transformation in der Medizin schreitet rasch voran. Mittlerweile zur Verfügung stehende technologische Entwicklungen, die bessere Behandlungen ermöglichen und aus der Sicht der Patientinnen und Patienten viele Vorteile mit sich bringen, etablieren sich.

Demgegenüber ist der effektive Schutz von Patientendaten hierbei nicht immer im Blick. Im Gegenteil: beim Einsatz digitaler Technologien im Gesundheitsbereich ist einer Reihe von Akteuren oftmals nicht hinreichend bewusst, welche Gefährdungen konkret für die Patientendaten bestehen, noch wie man diesen entgegentreten kann. Ereignisse wie der Hackerangriff auf
Daten von Personen des öffentlichen Lebens, der Anfang Januar 2019 öffentlich bekannt wurde, sowie aktuelle Presseberichte, nach denen Hacker-Angriffe auf Patientendaten sowie deren Handel im sog. Darknet deutlich zunehmen, belegen den Handlungsbedarf, der in diesem Zusammenhang besteht.

„Die aktuellen Vorkommnisse zeigen, dass wir als Gesellschaft insgesamt gefordert sind, auch im digitalen Zeitalter eine vertrauliche und sichere Gesundheitsversorgung zu gewährleisten." erklärt Prof. Dr. Dieter Kugelmann, Landesbeauftragter für den Datenschutz und die Informationsfreiheit Rheinland-Pfalz. „Allein die Vorteile der Digitalisierung in Anspruch zu nehmen, ohne die Risiken abzuwehren, die häufig ja den einzelnen Patienten persönlich treffen, wäre nicht nur rechtlich unzulässig, sondern aus meiner Sicht auch ethisch zu hinterfragen. Vorschlägen aus dem politischen Raum, den Datenschutz für Patienten zu lockern, um digitale Entwicklungen im Gesundheitssektor zu forcieren, trete ich deshalb grundsätzlich entgegen. Vielmehr sollte die Politik gemeinsam mit den Akteuren der Selbstverwaltung die Rahmenbedingungen für eine datenschutzgerechte digitale Gesundheitsversorgung aktiv gestalten und es nicht internationalen Unternehmen überlassen, hier unkontrolliert Standards zu setzen, die weder dem Patientenschutz noch dem Datenschutz gerecht werden.", so Kugelmann.

Mit dem Jahreswechsel 2018/19 hat Prof. Dr. Kugelmann für ein Jahr den Vorsitz der Datenschutzkonferenz (DSK), dem Gremium der unabhängigen deutschen Datenschutzaufsichtsbehörden des Bundes und der Länder, übernommen. Die Stärkung des Datenschutzes im Gesundheitsbereich gehört dabei zu seinen vorrangigen Anliegen.

Der Landesbeauftragte für den Datenschutz und die Informationsfreiheit Rheinland-Pfalz

Prof. Dr. Dieter Kugelmann 